Original Research Paper

\title{
Molecular Analysis of KRAS Mutation Associated with Colorectal Cancer in Iraqi Patients
}

\author{
${ }^{1}$ Rehab Subhi Ramadhan, ${ }^{1}$ Aola Sabah Ali and ${ }^{2}$ Rebah Najah Algafari \\ ${ }^{1}$ College of Biotechnology, Al-Nahrain University, Iraq \\ ${ }^{2}$ Biotechnology Research Center, Al-Nahrain University, Iraq
}

\author{
Article history \\ Received: 05-01-2018 \\ Revised: 03-02-2018 \\ Accepted: 3-03-2018 \\ Corresponding Author: \\ Rehab Subhi Ramadhan \\ College of Biotechnology, Al- \\ Nahrain University, Iraq \\ Email: rehabrebah2004@yahoo.com
}

\begin{abstract}
The role of KRAS gene was investigated in manifestation of colorectal cancer in Iraqi patients. A total 40 blood samples were collected during October 2016 to January 2017 from AL-Amal Hospital in Baghdad and 20 blood samples from healthy subjects served as control. Blood samples were collected from subjects of 40-70 years old for both patients and control. The study found that age group 61-70 years old were more susceptible to colorectal cancer with ratio of $40 \%$ more than younger individuals involved in this study with higher frequency in males with $75.5 \%$ than females who show $49.5 \%$ ( $p>0.01)$ when both compared to the same gender. DNA extracted from positive cancer samples and control were subjected to specific PCR amplification using 10 specifically designed primers for this study to amplify KRAS gene exons. DNA sequencing for the resulting amplicons showed the presence of significant genetic change that included substitution and insertion, causing $15 \%$ frame shift and $85 \%$ missense changes at positions 5920, consequently led to sever disruption in KRAS function.
\end{abstract}

Keywords: KRAS, Oncogenes, Colon Cancer, DNA Aberration

\section{Introduction}

Colorectal cancer represents $(15 \%)$ of worldwide malignancies and is the third cause of cancer in men $(10 \%)$ of total and the second cause of cancer in women after breast cancer (9.4\%) of total (Center et al., 2009). Colorectal cancer is a complex disease influenced by both genetic and environmental factors. Lifestyle and environmental risk factors include, for instance, smoking, diet and physical inactivity (Giovannucci, 2002; Botteri et al., 2008). Interestingly, physical inactivity has been estimated to cause up to $(10 \%)$ of the burden of Colorectal cancer (Lee et al., 2012). Environmental and lifestyle factors partly explain the high rate of colorectal cancer observed in the Western world. In addition, an increased risk for CRC has also been reported for individuals with Inflammatory Bowel Disease (IBD) (Dyson and Rutter, 2012). The development of colorectal cancer is a multistep process characterized by the accumulation of genetic alterations (Arteaga, 2002). Along the progression from normal colonic epithelial cells, small adenoma, advanced adenoma and finally to carcinoma, the KRAS oncogene mutation has a role in a significant proportion of CRCs. The KRAS gene encodes a $21-\mathrm{kDa}$ small protein that is activated transiently as a response to extracellular stimuli or signals such as growth factors, cytokines and hormones via cell surface receptors (Malumbres and Barbacid, 2003). The gene is located on the short arm of chromosome 12 (12p). It is mutated in (40-50\%) of sporadic cases of CRC. KRAS has been reported to be mutated in about $(30 \%)$ of colorectal adenomas and $(30 \%)$ to $(50 \%)$ of CRCs. Kirsten Rat Sarcoma viral oncogene homolog (KRAS) is an oncogene that encodes a small GTPase transduction protein called KRAS (Castagnola and Giaretti, 2005). The point mutations are seen in codon 12 in most cases but also at codon 13 and 61. It has been shown that KRAS mutations in metastatic disease are a predictor of resistance to Cetuximab (antiEGFR) therapy and, furthermore, they are associated with a worse prognosis of disease (Karapetis et al., 2008).

The aim of study was the detection the single nucleotide polymorphism in KRAS gene associated with incidence of colorectal cancer. 


\section{Materials and Methods}

Collection of Blood Samples: The blood samples were collected at Al-Amal hospital from 40 patients after they diagnosed with colorectal cancer using bio marker (CA19-9). Their ages ranged between (40-70) years as the majority of individuals attending the hospital for treatment. A volume of $5 \mathrm{~mL}$ of peripheral blood was collected by vein puncture and divided into two tubes, 1 $\mathrm{mL}$ to EDTA tube and store at $-20^{\circ} \mathrm{C}$ for DNA extraction. The $4 \mathrm{~mL}$ was transferred to a plain tube, for a biomarker test (CA19-9).

DNA extraction from blood: The gSYNCTM DNA Extraction Kit from Geneaid (Taiwan) was used for this purpose as instructed by the manufacturer. The extraction procedure mainly depends upon spin column technique which gave DNA purity of 1.8-2 and concentration of 80-120 ng/sample.

Primers used for DNA amplification: KRAS exons were amplified using the following primers designed in this study.

\begin{tabular}{|c|c|c|}
\hline $\begin{array}{l}\text { Primer } \\
\text { name }\end{array}$ & r Sequences $3 `---5$ & $\begin{array}{l}\text { Product } \\
\text { size }\end{array}$ \\
\hline \multirow[t]{2}{*}{1} & F GTCTCCCTGTGTCAGACTGC & 433 \\
\hline & R AATGTCTTGGCACACCACCA & \\
\hline \multirow{2}{*}{2} & F TCCCTGTGTCAGACTGCTCT & 313 \\
\hline & R AGGACCACCACAGAGTGAGA & \\
\hline \multirow[t]{2}{*}{3} & F AGGGACTAGGGCAGTTTGGA & 386 \\
\hline & R CACCTCACCATGCCATCTCA & \\
\hline \multirow[t]{2}{*}{4} & F TCTCCCTGTGTCAGACTGCT & 642 \\
\hline & R ACCTCACCATGCCATCTCAC & \\
\hline \multirow[t]{2}{*}{5} & F TGGTGGTGTGCCAAGACATT & 480 \\
\hline & R CCCAAGACTGGCACTGAAGA & \\
\hline \multirow[t]{2}{*}{6} & F CTTCCACATGCCCCATGACT & 370 \\
\hline & R ACTGTTACCAGGAGCAGTCC & \\
\hline \multirow[t]{2}{*}{7} & F TCTTGCCTCCCTACCTTCCA & 385 \\
\hline & R TACTGTTACCAGGAGCAGTCC & \\
\hline \multirow[t]{2}{*}{8} & F CCTCССТАССТТССАСАТGC & 378 \\
\hline & R CTGTTACCAGGAGCAGTCCTA & \\
\hline \multirow[t]{2}{*}{9} & F ACTGTTGTCACCATTGCACA & 734 \\
\hline & R TGTGCATGTTTCAGTTTACACTAT & \\
\hline \multirow[t]{2}{*}{10} & F GTTTGAAGTGCCTGTTTGGGA & 324 \\
\hline & R CACAAAAGAAAGCACAATGTACAAA & \\
\hline
\end{tabular}

\section{DNA Amplification Programs and PCR Conditions}

Because of the different melting temperatures for each set of primer, the following conditions were used for optimum results:

Primers 1,7 and 8

\begin{tabular}{lll}
\hline Thermal cycler protocol & No. of cycle & Temperature -time \\
\hline Initial Denaturation & 1 cycle & $94^{\circ} \mathrm{C}$ for $5 \mathrm{~min}$ \\
Denaturation & 35 cycle & $94^{\circ} \mathrm{C}$ for $1 \mathrm{~min}$ \\
Annealing & & $59^{\circ} \mathrm{C}$ for $1 \mathrm{~min}$ \\
Extension & & $72^{\circ} \mathrm{C}$ for $1 \mathrm{~min}$ \\
Final extension & 1 cycle & $72^{\circ} \mathrm{C}$ for $10 \mathrm{~min}$ \\
\hline
\end{tabular}

Primers $2,3,4,5$ and 6

\begin{tabular}{lll}
\hline Thermal cycler protocol & No. of cycle & Temperature -time \\
\hline Initial Denaturation & 1 cycle & $94^{\circ} \mathrm{C}$ for $5 \mathrm{~min}$ \\
Denaturation & 35 cycle & $94^{\circ} \mathrm{C}$ for $1 \mathrm{~min}$ \\
Annealing & & $56^{\circ} \mathrm{C}$ for $1 \mathrm{~min}$ \\
Extension & & $72^{\circ} \mathrm{C}$ for $1 \mathrm{~min}$ \\
Final Extension & 1 cycle & $72^{\circ} \mathrm{C}$ for $10 \mathrm{~min}$ \\
\hline
\end{tabular}

Primers 9 and 10

\begin{tabular}{lll}
\hline Thermal cycler protocol & No. of cycle & Temperature-time \\
\hline Initial Denaturation & 1 cycle & $94^{\circ} \mathrm{C}$ for $5 \mathrm{~min}$ \\
Denaturation & 35 cycle & $94^{\circ} \mathrm{C}$ for $1 \mathrm{~min}$ \\
Annealing & & $53^{\circ} \mathrm{C}$ for $1 \mathrm{~min}$ \\
Extension & & $72^{\circ} \mathrm{C}$ for $1 \mathrm{~min}$ \\
Final extension & 1 cycle & $72^{\circ} \mathrm{C}$ for $10 \mathrm{~min}$ \\
\hline
\end{tabular}

\section{Electrophoresis Conditions}

The resulting PCR products were subjected to electrophoresis using $2 \%$ agarose at $10 \mathrm{v} / \mathrm{cm}$ field strength for $1 \mathrm{~h}$ and photographed using Biorad gel documentation system.

\section{Statistical analYsis}

The Statistical Analysis System-SAS (2012) program was used to signify the relation among age, gender and colorectal cancer manifestation.

\section{Results}

In this study, forty blood samples were collected from patients suffering from colorectal cancer and 20 healthy (control), with age ranging from 40 to 70 years. Chi-square tests was used to establish the relation between age and gender. Results are shown in Table 1.

Colorectal cancer was found to be more frequent in male than female as shown in Table 2.

\section{Molecular Analysis of KRAS}

Ten primers were designed to amplify all KRAS exons to be used for sequencing and subsequent analysis. Results showed that there is a significant change in DNA sequence among patients and as shown in Fig. 1.

Further details of genetic changes are listed in Table 3.

In almost all patients subjected to DNA analysis of KRAS gene, genetic change included missense (83 SNPs) mutation with less frequency of frame-shift (2 SNPs) resulting in deformed protein and non functional enzyme.

Table 1: Distribution of patients according to age group

\begin{tabular}{lll}
\hline Age group (years) & Number of patients & Percentage (\%) \\
\hline $40-50$ & 10 & 25 \\
$51-60$ & 14 & 35 \\
$61-70$ & 16 & 40 \\
Total & 40 & 100 \\
Chi-square value $\left(\mathrm{x}^{2}\right)$ & - & $9.261 * *$ \\
\hline
\end{tabular}



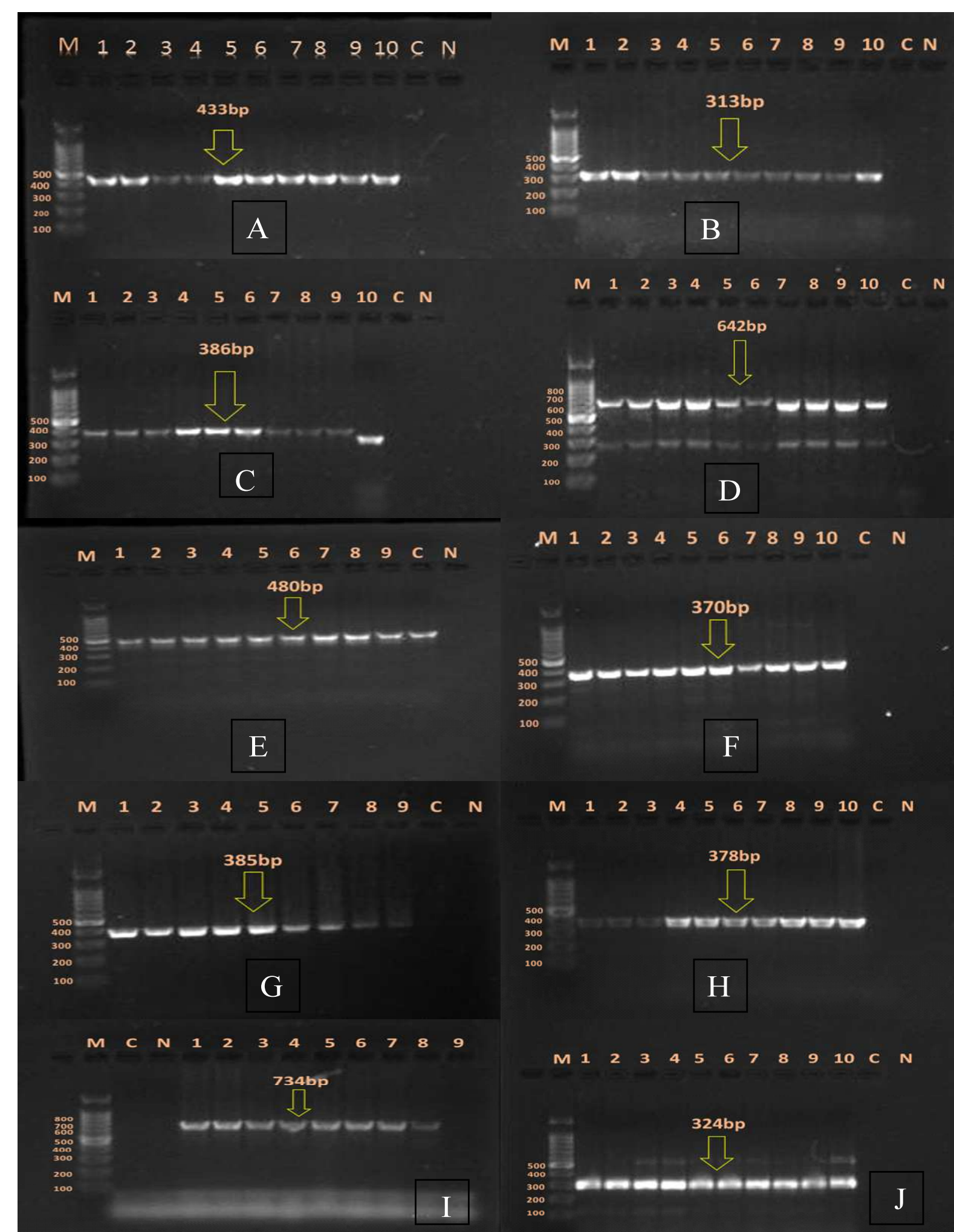

$\begin{array}{lllllllllllll}M & 1 & 2 & 3 & 4 & 5 & 6 & 7 & 8 & 9 & 10 & C & N\end{array}$
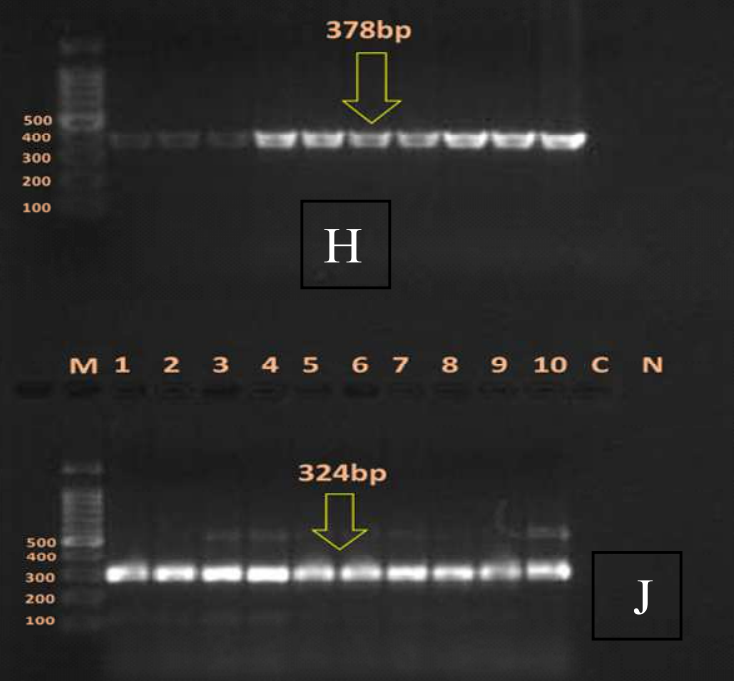

Fig. 1:KRAS specific amplification using primers designed in this study. Designations A, B, C, D, E, F, G, H, I and J represent primers, $1,2,3,4,5,6,7,8,9$ and 10 respectively. $\mathrm{M}$ is $100 \mathrm{bp}$ DNA marker, whereas $\mathrm{C}$ and $\mathrm{N}$ are control negative and normal DNA respectively. Patients are designated with numbers from 1-10 


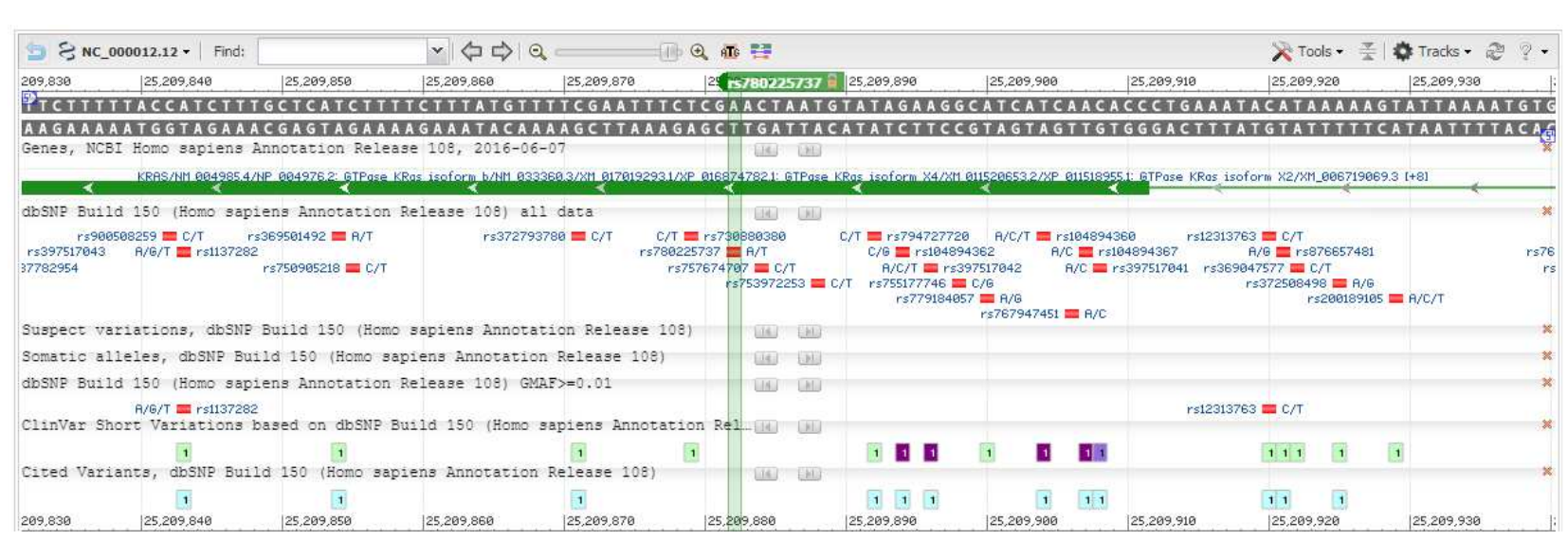

Fig. 2: Mapping the genetic change in KRAS gene. The figure shows Graphical distribution of KRAS SNPs in the sequence obtained from colorectal cancer patients. The figure also elaborates amino acid change resulted from the SNP which resulted in pathogenic effect (red dots) and purple squares, whereas light blue squares represent non effective SNPs. The figure was generated using NCBI analysis tool (https://www.ncbi.nlm.nih.gov/protein/NP_203524.1?report=graph\&v=1:63\&content=5\&m=13)

Table 2: Distribution of patients according to gender

\begin{tabular}{lllc}
\hline Gender & Number of patients & Percentage $(\%)$ & Chi-square value $\left(\mathrm{x}^{2}\right)$ \\
\hline Male & 23 & 57.50 & $10.974^{* *}$ \\
Female & 17 & 42.50 & $10.052^{* *}$ \\
Total & 40 & $100 \%$ & $9.261^{* *}$
\end{tabular}

$* *(\mathrm{P}<0.01)$

Table 3: SNP linked to Gene KRAS Via Contig Annotation. The table was generated using The SNP GeneView for human variation depending on the sequence obtained in this study. The tool used for analysis is available at https://www.ncbi.nlm.nih.gov/ analysis tools

\begin{tabular}{|c|c|c|c|c|c|c|c|c|c|}
\hline $\begin{array}{l}\text { Chr. } \\
\text { position }\end{array}$ & $\begin{array}{l}\text { mRNA } \\
\text { pos }\end{array}$ & $\begin{array}{l}\text { dbSNP rs\# } \\
\text { cluster id }\end{array}$ & $\begin{array}{l}\text { Hetero- } \\
\text { zygosity }\end{array}$ & $\begin{array}{l}\text { Clinical } \\
\text { Significance }\end{array}$ & Function & $\begin{array}{l}\text { dbSNP } \\
\text { allele }\end{array}$ & $\begin{array}{l}\text { Protein } \\
\text { residue }\end{array}$ & $\begin{array}{l}\text { Codon } \\
\text { pos }\end{array}$ & $\begin{array}{l}\text { Amino acid } \\
\text { pos }\end{array}$ \\
\hline \multirow[t]{2}{*}{25209808} & 746 & rs775000854 & 0.000 & & Missense & A & Tyr [Y] & 2 & 185 \\
\hline & & & & & Contig reference & G & Cys [C] & 2 & 185 \\
\hline 25209822 & 730 & rs749587181 & 0.000 & & & - & & 1 & 180 \\
\hline \multirow[t]{2}{*}{25209822} & 732 & rs 762532538 & 0.000 & & Missense & $\mathrm{C}$ & Asn $[N]$ & 3 & 180 \\
\hline & & & & & Contig reference & $\mathrm{G}$ & Lys $[\mathrm{K}]$ & 3 & 180 \\
\hline \multirow[t]{2}{*}{25209826} & 728 & rs763736188 & 0.000 & & Missense & G & $\operatorname{Arg}[R]$ & 2 & 179 \\
\hline & & & & & Contig reference & A & Lys $[\mathrm{K}]$ & 2 & 179 \\
\hline 25209828 & 725 & rs587782954 & N.D. & Uncertain significance & & GAA & Lys [K] & 2 & 180 \\
\hline \multirow{2}{*}{25209828} & 726 & rs751233524 & N.D. & & Synonymous & G & Lys $[\mathrm{K}]$ & 3 & 178 \\
\hline & & & & & Contig reference & A & Lys $[\mathrm{K}]$ & 3 & 178 \\
\hline 25209829 & 723 & rs397517043 & 0.001 & Uncertain significance & & - & & 3 & 180 \\
\hline \multirow[t]{2}{*}{25209842} & 712 & rs900508259 & N.D. & & Missense & A & Ser $[S]$ & 1 & 174 \\
\hline & & & & & Contig reference & $\mathrm{G}$ & Gly [G] & 1 & 174 \\
\hline \multirow[t]{2}{*}{25209843} & 711 & rs1137282 & 0.316 & Likely benign & Synonymous & $\mathrm{C}$ & Asp [D] & 3 & 173 \\
\hline & & & & & Contig reference & $\mathrm{T}$ & Asp [D] & 3 & 173 \\
\hline \multirow[t]{2}{*}{25209854} & 700 & rs369501492 & 0.000 & other & Missense & $\mathrm{T}$ & Leu $[\mathrm{L}]$ & 1 & 170 \\
\hline & & & & & Contig reference & A & $\operatorname{Met}[\mathrm{M}]$ & 1 & 170 \\
\hline \multirow[t]{2}{*}{25209855} & 699 & rs750905218 & 0.000 & & Synonymous & A & Lys [K] & 3 & 169 \\
\hline & & & & & Contig reference & G & Lys $[\mathrm{K}]$ & 3 & 169 \\
\hline \multirow[t]{2}{*}{25209871} & 683 & rs372793780 & 0.000 & Uncertain significance & Missense & A & Gln [Q] & 2 & 164 \\
\hline & & & & & Contig reference & $\mathrm{G}$ & $\operatorname{Arg}[R]$ & 2 & 164 \\
\hline \multirow[t]{2}{*}{25209879} & 675 & rs730880380 & N.D. & Benign & Synonymous & $\mathrm{G}$ & $\operatorname{Arg}[R]$ & 3 & 161 \\
\hline & & & & & Contig reference & A & $\operatorname{Arg}[R]$ & 3 & 161 \\
\hline \multirow[t]{2}{*}{25209882} & 672 & rs780225737 & 0.000 & & Synonymous & A & Val [V] & 3 & 160 \\
\hline & & & & & Contig reference & $\mathrm{T}$ & Val [V] & 3 & 160 \\
\hline \multirow[t]{2}{*}{25209884} & 670 & rs757674707 & N.D. & & Missense & A & Ile $[1]$ & 1 & 160 \\
\hline & & & & & Contig reference & $\mathrm{G}$ & Val [V] & 1 & 160 \\
\hline \multirow[t]{2}{*}{25209888} & 666 & rs753972253 & 0.000 & & Synonymous & $\mathrm{G}$ & $\operatorname{Thr}[\mathrm{T}]$ & 3 & 158 \\
\hline & & & & & Contig reference & A & $\operatorname{Thr}[\mathrm{T}]$ & 3 & 158 \\
\hline \multirow[t]{2}{*}{25209892} & 662 & rs794727720 & N.D. & Uncertain significance & Missense & $\mathrm{G}$ & Cys [C] & 2 & 157 \\
\hline & & & & & Contig reference & A & Tyr $[Y]$ & 2 & 157 \\
\hline
\end{tabular}


Rehab Subhi Ramadhan et al. / American Journal of Biochemistry and Biotechnology 2018, 14 (1): 48.56 DOI: $10.3844 /$ ajbbsp.2018.48.56

\begin{tabular}{|c|c|c|c|c|c|c|c|c|c|}
\hline \multirow{2}{*}{25209894} & \multirow[t]{2}{*}{660} & \multirow{2}{*}{ rs104894362 } & \multirow[t]{2}{*}{ N.D. } & \multirow{2}{*}{ Pathogenic } & Missense & $\mathrm{G}$ & Leu [L] & 3 & 156 \\
\hline & & & & & Contig reference & $\mathrm{C}$ & Phe [F] & 3 & 156 \\
\hline \multirow[t]{3}{*}{25209896} & \multirow[t]{3}{*}{658} & \multirow[t]{3}{*}{ rs397517042 } & \multirow[t]{3}{*}{ N.D. } & \multirow[t]{3}{*}{ Pathogenic } & Missense & A & Ile $[\mathrm{I}]$ & 1 & 156 \\
\hline & & & & & Missense & G & $\mathrm{Val}[\mathrm{V}]$ & 1 & 156 \\
\hline & & & & & Contig reference & $\mathrm{T}$ & Phe $[F]$ & 1 & 156 \\
\hline 25209898 & 656 & rs755177746 & 0.000 & & Missense & G & Gly [G] & 2 & 155 \\
\hline & & & & & Contig reference & $\mathrm{C}$ & Ala [A] & 2 & 155 \\
\hline 25209900 & 654 & rs779184057 & 0.000 & Uncertain significance & Synonymous & $\mathrm{C}$ & Asp [D] & 3 & 154 \\
\hline & & & & & Contig reference & $\mathrm{T}$ & Asp [D] & 3 & 154 \\
\hline 25209904 & 650 & rs 104894360 & N.D. & Pathogenic & Missense & G & Gly [G] & 2 & 153 \\
\hline & & & & & Missense & $\mathrm{T}$ & Val [V] & 2 & 153 \\
\hline & & & & & Contig reference & A & Asp [D] & 2 & 153 \\
\hline 25209906 & 648 & rs767947451 & 0.000 & & Synonymous & G & Val [V] & 3 & 152 \\
\hline & & & & & Contig reference & $\mathrm{T}$ & $\mathrm{Val}[\mathrm{V}]$ & 3 & 152 \\
\hline 25209907 & 647 & rs104894367 & N.D. & Pathogenic & Missense & $\mathrm{G}$ & Gly [G] & 2 & 152 \\
\hline & & & & & Contig reference & $\mathrm{T}$ & Val [V] & 2 & 152 \\
\hline 25209908 & 646 & rs397517041 & N.D. & Likely pathogenic & Missense & $\mathrm{T}$ & Phe $[\mathrm{F}]$ & 1 & 152 \\
\hline & & & & & Contig reference & G & Val [V] & 1 & 152 \\
\hline 25225620 & 636 & rs777364720 & N.D. & & Synonymous & G & $\operatorname{Thr}[\mathrm{T}]$ & 3 & 148 \\
\hline & & & & & Contig reference & A & $\operatorname{Thr}[\mathrm{T}]$ & 3 & 148 \\
\hline 25225625 & 631 & rs387907206 & N.D. & Pathogenic & Missense & G & Glu [E] & 1 & 147 \\
\hline & & & & & Contig reference & A & Lys [K] & 1 & 147 \\
\hline 25225628 & 628 & rs121913527 & N.D. & Uncertain significance & Missense & A & $\operatorname{Thr}[\mathrm{T}]$ & 1 & 146 \\
\hline & & & & & Contig reference & G & $\mathrm{Ala}[\mathrm{A}]$ & 1 & 146 \\
\hline 25225632 & 624 & rs766109434 & 0.000 & & Synonymous & G & $\operatorname{Thr}[\mathrm{T}]$ & 3 & 144 \\
\hline & & & & & Contig reference & A & $\operatorname{Thr}[\mathrm{T}]$ & 3 & 144 \\
\hline 25225641 & 615 & rs138669124 & 0.000 & & Missense & A & Leu [L] & 3 & 141 \\
\hline & & & & & Contig reference & $\mathrm{T}$ & Phe $[\mathrm{F}]$ & 3 & 141 \\
\hline 25225651 & 605 & rs754870563 & 0.000 & & Missense & A & Glu [E] & 2 & 138 \\
\hline & & & & & Contig reference & G & Gly [G] & 2 & 138 \\
\hline 25225652 & 604 & rs778702415 & 0.000 & & Missense & A & $\operatorname{Arg}[R]$ & 1 & 138 \\
\hline & & & & & Contig reference & G & Gly [G] & 1 & 138 \\
\hline 25225653 & 603 & rs752731198 & 0.000 & & Synonymous & $\mathrm{C}$ & Tyr [Y] & 3 & 137 \\
\hline & & & & & Contig reference & $\mathrm{T}$ & Tyr [Y] & 3 & 137 \\
\hline 25225657 & 599 & rs757816355 & 0.000 & & Missense & A & Asn $[N]$ & 2 & 136 \\
\hline & & & & & Contig reference & G & Ser $[\mathrm{S}]$ & 2 & 136 \\
\hline 25225663 & 593 & rs373500216 & 0.000 & & Missense & G & Gly [G] & 2 & 134 \\
\hline & & & & & Contig reference & $\mathrm{C}$ & Ala $[\mathrm{A}]$ & 2 & 134 \\
\hline 25225675 & 581 & rs730880473 & 0.000 & Uncertain significance & Missense & $\mathrm{T}$ & Val [V] & 2 & 130 \\
\hline & & & & & Contig reference & $\mathrm{C}$ & Ala $[\mathrm{A}]$ & 2 & 130 \\
\hline 25225681 & 575 & rs746609817 & 0.000 & & Missense & $\mathrm{G}$ & $\operatorname{Arg}[R]$ & 2 & 128 \\
\hline & & & & & Contig reference & A & Lys $[\mathrm{K}]$ & 2 & 128 \\
\hline 25225683 & 573 & rs770720889 & 0.000 & & Synonymous & G & $\operatorname{Thr}[\mathrm{T}]$ & 3 & 127 \\
\hline & & & & & Contig reference & A & $\operatorname{Thr}[\mathrm{T}]$ & 3 & 127 \\
\hline 25225684 & 572 & rs781634879 & 0.000 & & Missense & G & $\operatorname{Arg}[R]$ & 2 & 127 \\
\hline & & & & & Contig reference & $\mathrm{C}$ & Thr [T] & 2 & 127 \\
\hline 25225694 & 562 & rs575569675 & 0.000 & & Missense & $\mathrm{T}$ & Ser $[S]$ & 1 & 124 \\
\hline & & & & & Contig reference & A & $\operatorname{Thr}[\mathrm{T}]$ & 1 & 124 \\
\hline 25225709 & 547 & rs730880471 & N.D. & Likely pathogenic & Missense & A & Asn $[\mathrm{N}]$ & 1 & 119 \\
\hline & & & & & Contig reference & G & Asp [D] & 1 & 119 \\
\hline 25225713 & 543 & rs770248150 & 0.000 & & Missense & $\mathrm{C}$ & Asn $[N]$ & 3 & 117 \\
\hline & & & & & Contig reference & A & Lys [K] & 3 & 117 \\
\hline 25225717 & 539 & rs202247812 & N.D. & untested & Missense & G & Ser $[S]$ & 2 & 116 \\
\hline & & & & & Contig reference & A & $\operatorname{Asn}[\mathrm{N}]$ & 2 & 116 \\
\hline 25225730 & 526 & rs775836436 & 0.000 & & Missense & A & Ile [I] & 1 & 112 \\
\hline & & & & & Contig reference & G & $\mathrm{Val}[\mathrm{V}]$ & 1 & 112 \\
\hline 25225742 & 514 & rs763553461 & 0.000 & & Missense & $\mathrm{T}$ & Tyr [Y] & 1 & 108 \\
\hline & & & & & Contig reference & G & Asp [D] & 1 & 108 \\
\hline 25227234 & 482 & rs727503106 & N.D. & Likely pathogenic & Missense & A & Lys [K] & 2 & 97 \\
\hline & & & & & Contig reference & G & $\operatorname{Arg}[R]$ & 2 & 97 \\
\hline 25227260 & 456 & rs370920665 & 0.000 & Uncertain significance & Synonymous & G & Lys $[\mathrm{K}]$ & 3 & 88 \\
\hline & & & & & Contig reference & A & Lys [K] & 3 & 88 \\
\hline 25227262 & 454 & rs953088090 & N.D. & & Missense & G & Glu [E] & 1 & 88 \\
\hline & & & & & Contig reference & A & Lys [K] & 1 & 88 \\
\hline 25227263 & 453 & rs397517038 & N.D. & Uncertain significance & Frame shift & - & Asn $[N]$ & 3 & 88 \\
\hline & & & & & Contig reference & $\mathrm{T}$ & Lys $[\mathrm{K}]$ & 3 & 88 \\
\hline 25227275 & 441 & rs751117590 & 0.000 & & Synonymous & $\mathrm{T}$ & Ala $[\mathrm{A}]$ & 3 & 83 \\
\hline & & & & & Contig reference & $\mathrm{C}$ & $\mathrm{Ala}[\mathrm{A}]$ & 3 & 83 \\
\hline
\end{tabular}


Rehab Subhi Ramadhan et al. / American Journal of Biochemistry and Biotechnology 2018, 14 (1): 48.56 DOI: $10.3844 /$ ajbbsp.2018.48.56

\begin{tabular}{|c|c|c|c|c|c|c|c|c|c|}
\hline \multirow[t]{2}{*}{25227288} & 428 & rs868857258 & N.D. & & Missense & $\mathrm{C}$ & Pro $[\mathrm{P}]$ & 2 & 79 \\
\hline & & & & & Contig reference & $\mathrm{T}$ & Leu [L] & 2 & 79 \\
\hline \multirow[t]{2}{*}{25227294} & 422 & rs756890312 & 0.000 & & Missense & $\mathrm{C}$ & Ala $[\mathrm{A}]$ & 2 & 77 \\
\hline & & & & & Contig reference & $\mathrm{G}$ & Gly [G] & 2 & 77 \\
\hline \multirow[t]{2}{*}{25227296} & 420 & rs 727503107 & N.D. & Uncertain significance & Synonymous & $\mathrm{A}$ & Glu [E] & 3 & 76 \\
\hline & & & & & Contig reference & G & Glu [E] & 3 & 76 \\
\hline \multirow[t]{2}{*}{25227300} & 416 & rs780974222 & 0.000 & & Missense & $\mathrm{C}$ & Ala [A] & 2 & 75 \\
\hline & & & & & Contig reference & G & Gly [G] & 2 & 75 \\
\hline \multirow[t]{2}{*}{25227302} & 414 & rs745580373 & 0.000 & & Synonymous & $\mathrm{C}$ & Thr $[\mathrm{T}]$ & 3 & 74 \\
\hline & & & & & Contig reference & $\mathrm{T}$ & Thr [T] & 3 & 74 \\
\hline \multirow[t]{2}{*}{25227304} & 412 & rs770020203 & 0.000 & & Missense & $\mathrm{G}$ & Ala $[\mathrm{A}]$ & 1 & 74 \\
\hline & & & & & Contig reference & $\mathrm{A}$ & Thr [T] & 1 & 74 \\
\hline \multirow[t]{2}{*}{25227305} & 411 & rs 104886027 & N.D. & untested & Synonymous & $\mathrm{A}$ & $\operatorname{Arg}[\mathrm{R}]$ & 3 & 73 \\
\hline & & & & & Contig reference & G & $\operatorname{Arg}[R]$ & 3 & 73 \\
\hline \multirow[t]{2}{*}{25227308} & 408 & rs 104886028 & N.D. & untested & Missense & $\mathrm{A}$ & Ile $[\mathrm{I}]$ & 3 & 72 \\
\hline & & & & & Contig reference & $\mathrm{G}$ & Met $[\mathrm{M}]$ & 3 & 72 \\
\hline \multirow[t]{2}{*}{25227310} & 406 & rs727504662 & N.D. & Pathogenic & Missense & $\mathrm{T}$ & Leu [L] & 1 & 72 \\
\hline & & & & & Contig reference & A & Met [M] & 1 & 72 \\
\hline \multirow[t]{6}{*}{25227313} & 403 & rs387907205 & N.D. & Likely pathogenic & Missense & $\mathrm{C}$ & His $[\mathrm{H}]$ & 1 & 71 \\
\hline & & & & & Missense & $\mathrm{C}$ & His $[\mathrm{H}]$ & 1 & 71 \\
\hline & & & & & Missense & $\mathrm{G}$ & Asp [D] & 1 & 71 \\
\hline & & & & & Missense & G & Asp [D] & 1 & 71 \\
\hline & & & & & Contig reference & $\mathrm{T}$ & Tyr [Y] & 1 & 71 \\
\hline & & & & & Contig reference & $\mathrm{T}$ & Tyr [Y] & 1 & 71 \\
\hline 25227314 & 402 & rs780492744 & 0.000 & & Synonymous & $\mathrm{A}$ & Gln [Q] & 3 & 70 \\
\hline & & & & & Contig reference & $\mathrm{G}$ & Gln [Q] & 3 & 70 \\
\hline 25227326 & 390 & rs200229810 & 0.000 & Uncertain significance & Synonymous & $\mathrm{G}$ & Ala [A] & 3 & 66 \\
\hline & & & & & Contig reference & A & Ala $[\mathrm{A}]$ & 3 & 66 \\
\hline 25227335 & 379 & rs730880469 & N.D. & Likely pathogenic & & - & & 1 & 63 \\
\hline 25227341 & 375 & rs 17851045 & N.D. & Pathogenic & Missense & $\mathrm{C}$ & His $[\mathrm{H}]$ & 3 & 61 \\
\hline & & & & & Missense & $\mathrm{T}$ & His $[\mathrm{H}]$ & 3 & 61 \\
\hline & & & & & Contig reference & $\mathrm{A}$ & Gln [Q] & 3 & 61 \\
\hline 25227342 & 374 & rs 121913240 & N.D. & Pathogenic & Missense & $\mathrm{C}$ & Pro $[\mathrm{P}]$ & 2 & 61 \\
\hline & & & & & Missense & $\mathrm{G}$ & $\operatorname{Arg}[\mathrm{R}]$ & 2 & 61 \\
\hline & & & & & Missense & $\mathrm{T}$ & Leu [L] & 2 & 61 \\
\hline & & & & & Contig reference & $\mathrm{A}$ & Gln [Q] & 2 & 61 \\
\hline 25227343 & 373 & rs121913238 & N.D. & Pathogenic & Missense & $\mathrm{A}$ & Lys [K] & 1 & 61 \\
\hline & & & & & Missense & $\mathrm{G}$ & Glu [E] & 1 & 61 \\
\hline & & & & & Contig reference & $\mathrm{C}$ & Gln [Q] & 1 & 61 \\
\hline 25227344 & 372 & rs397517037 & N.D. & Uncertain significance & Synonymous & A & Gly [G] & 3 & 60 \\
\hline & & & & & Contig reference & $\mathrm{T}$ & Gly [G] & 3 & 60 \\
\hline 25227345 & 371 & rs 727503108 & N.D. & Pathogenic & Missense & $\mathrm{T}$ & Val [V] & 2 & 60 \\
\hline & & & & & Contig reference & $\mathrm{G}$ & Gly [G] & 2 & 60 \\
\hline 25227346 & 370 & rs104894359 & N.D. & Pathogenic & Missense & $\mathrm{A}$ & $\operatorname{Ser}[\mathrm{S}]$ & 1 & 60 \\
\hline & & & & & Missense & $\mathrm{C}$ & $\operatorname{Arg}[\mathrm{R}]$ & 1 & 60 \\
\hline & & & & & Contig reference & $\mathrm{G}$ & Gly [G] & 1 & 60 \\
\hline 25227348 & 368 & rs 104886029 & N.D. & untested & Missense & $\mathrm{T}$ & Val [V] & 2 & 59 \\
\hline & & & & & Contig reference & $\mathrm{C}$ & Ala $[\mathrm{A}]$ & 2 & 59 \\
\hline 25227349 & 367 & rs121913528 & N.D. & Likely pathogenic & Missense & $\mathrm{A}$ & Thr [T] & 1 & 59 \\
\hline & & & & & Missense & $\mathrm{A}$ & Thr [T] & 1 & 59 \\
\hline & & & & & Missense & $\mathrm{T}$ & Ser $[S]$ & 1 & 59 \\
\hline & & & & & Missense & $\mathrm{T}$ & Ser $[S]$ & 1 & 59 \\
\hline & & & & & Contig reference & $\mathrm{G}$ & Ala $[\mathrm{A}]$ & 1 & 59 \\
\hline & & & & & Contig reference & G & Ala $[\mathrm{A}]$ & 1 & 59 \\
\hline 25227351 & 365 & rs104894364 & N.D. & Pathogenic & Missense & $\mathrm{T}$ & Ile [I] & 2 & 58 \\
\hline & & & & & Contig reference & $\mathrm{C}$ & Thr [T] & 2 & 58 \\
\hline 25227356 & 360 & rs958790148 & N.D. & & Synonymous & $\mathrm{T}$ & Leu [L] & 3 & 56 \\
\hline & & & & & Contig reference & $\mathrm{C}$ & Leu [L] & 3 & 56 \\
\hline 25227374 & 342 & rs774909024 & 0.000 & & Synonymous & $\mathrm{G}$ & Thr [T] & 3 & 50 \\
\hline & & & & & Contig reference & $\mathrm{C}$ & Thr [T] & 3 & 50 \\
\hline 25227376 & 340 & rs730880470 & N.D. & Uncertain significance & Missense & $\mathrm{T}$ & Ser $[S]$ & 1 & 50 \\
\hline & & & & & Contig reference & A & $\operatorname{Thr}[\mathrm{T}]$ & 1 & 50 \\
\hline 25227386 & 330 & rs 904755552 & N.D. & & Missense & G & $\operatorname{Met}[\mathrm{M}]$ & 3 & 46 \\
\hline & & & & & Contig reference & $\mathrm{T}$ & Ile $[\mathrm{I}]$ & 3 & 46 \\
\hline 25245277 & 300 & rs 727503109 & N.D. & Likely pathogenic & Missense & G & Met [M] & 3 & 36 \\
\hline & & & & & Contig reference & A & Ile [I] & 3 & 36 \\
\hline 25245284 & 293 & rs 104894366 & N.D. & Pathogenic & Missense & G & $\operatorname{Arg}[R]$ & 2 & 34 \\
\hline & & & & & Missense & $\mathrm{T}$ & Leu [L] & 2 & 34 \\
\hline & & & & & Contig reference & $\mathrm{C}$ & Pro [P] & 2 & 34 \\
\hline
\end{tabular}


Rehab Subhi Ramadhan et al. / American Journal of Biochemistry and Biotechnology 2018, 14 (1): 48.56 DOI: 10.3844/ajbbsp.2018.48.56

\begin{tabular}{|c|c|c|c|c|c|c|c|c|c|}
\hline \multirow[t]{2}{*}{25245292} & 285 & rs377354475 & 0.000 & Uncertain significance & Synonymous & G & Glu [E] & 3 & 31 \\
\hline & & & & & Contig reference & $\mathrm{A}$ & Glu [E] & 3 & 31 \\
\hline \multirow[t]{2}{*}{25245295} & 282 & rs113623140 & 0.001 & Benign & Synonymous & $\mathrm{T}$ & Asp [D] & 3 & 30 \\
\hline & & & & & Contig reference & $\mathrm{C}$ & Asp [D] & 3 & 30 \\
\hline \multirow[t]{2}{*}{25245309} & 268 & rs 794727277 & N.D. & Uncertain significance & Missense & $\mathrm{T}$ & Tyr [Y] & 1 & 26 \\
\hline & & & & & Contig reference & $\mathrm{A}$ & $\operatorname{Asn}[\mathrm{N}]$ & 1 & 26 \\
\hline \multirow[t]{2}{*}{25245312} & 264 & rs754767487 & 0.000 & & Frame shift & $\mathrm{T}$ & $\operatorname{Ser}[S]$ & 3 & 25 \\
\hline & & & & & Contig reference & - & $\mathrm{G} \ln [\mathrm{Q}]$ & 3 & 25 \\
\hline \multirow[t]{2}{*}{25245317} & 260 & rs730880472 & N.D. & Likely pathogenic & Missense & $\mathrm{G}$ & Arg [R] & 2 & 23 \\
\hline & & & & & Contig reference & $\mathrm{T}$ & Leu [L] & 2 & 23 \\
\hline \multirow[t]{3}{*}{25245320} & 257 & rs727503110 & N.D. & Pathogenic & Missense & $\mathrm{G}$ & $\operatorname{Arg}[R]$ & 2 & 22 \\
\hline & & & & & Missense & $\mathrm{T}$ & Leu [L] & 2 & 22 \\
\hline & & & & & Contig reference & $\mathrm{A}$ & Gln [Q] & 2 & 22 \\
\hline \multirow[t]{2}{*}{25245321} & 256 & rs121913236 & N.D. & & Missense & A & Lys [K] & 1 & 22 \\
\hline & & & & & Contig reference & $\mathrm{C}$ & Gln [Q] & 1 & 22 \\
\hline \multirow[t]{2}{*}{25245325} & 252 & rs 747140926 & 0.000 & & Synonymous & $\mathrm{A}$ & Thr [T] & 3 & 20 \\
\hline & & & & & Contig reference & G & Thr [T] & 3 & 20 \\
\hline \multirow[t]{3}{*}{25245328} & 249 & rs 121913538 & N.D. & Uncertain significance & Missense & $\mathrm{C}$ & Phe $[\mathrm{F}]$ & 3 & 19 \\
\hline & & & & & Missense & $\mathrm{T}$ & Phe $[\mathrm{F}]$ & 3 & 19 \\
\hline & & & & & Contig reference & $\mathrm{G}$ & Leu [L] & 3 & 19 \\
\hline \multirow[t]{2}{*}{25245330} & 247 & rs 771188508 & 0.000 & & Synonymous & $\mathrm{C}$ & Leu [L] & 1 & 19 \\
\hline & & & & & Contig reference & $\mathrm{T}$ & Leu [L] & 1 & 19 \\
\hline \multirow[t]{2}{*}{25245334} & 243 & rs776785730 & 0.000 & & Synonymous & $\mathrm{C}$ & Ser $[S]$ & 3 & 17 \\
\hline & & & & & Contig reference & $\mathrm{T}$ & Ser [S] & 3 & 17 \\
\hline \multirow[t]{2}{*}{25245345} & 232 & rs104894365 & 0.000 & Pathogenic & Missense & A & Ile $[\mathrm{I}]$ & 1 & 14 \\
\hline & & & & & Contig reference & G & Val [V] & 1 & 14 \\
\hline \multirow[t]{3}{*}{25245346} & 231 & rs397517040 & 0.000 & Likely pathogenic & Synonymous & $\mathrm{A}$ & Gly [G] & 3 & 13 \\
\hline & & & & & Synonymous & $\mathrm{T}$ & Gly [G] & 3 & 13 \\
\hline & & & & & Contig reference & $\mathrm{C}$ & Gly [G] & 3 & 13 \\
\hline \multirow[t]{2}{*}{25245347} & 230 & rs112445441 & N.D. & Pathogenic & Missense & $\mathrm{A}$ & Asp [D] & 2 & 13 \\
\hline & & & & & Contig reference & G & Gly [G] & 2 & 13 \\
\hline \multirow[t]{4}{*}{25245348} & 229 & rs121913535 & N.D. & Pathogenic & Missense & $\mathrm{A}$ & $\operatorname{Ser}[S]$ & 1 & 13 \\
\hline & & & & & Missense & $\mathrm{C}$ & $\operatorname{Arg}[\mathrm{R}]$ & 1 & 13 \\
\hline & & & & & Missense & $\mathrm{T}$ & Cys [C] & 1 & 13 \\
\hline & & & & & Contig reference & $\mathrm{G}$ & Gly [G] & 1 & 13 \\
\hline \multirow[t]{2}{*}{25245350} & 227 & rs121913529 & 0.000 & Pathogenic & Missense & $\mathrm{A}$ & Asp [D] & 2 & 12 \\
\hline & & & & & Contig reference & G & Gly [G] & 2 & 12 \\
\hline \multirow[t]{4}{*}{25245351} & 226 & rs121913530 & 0.000 & Pathogenic & Missense & $\mathrm{A}$ & $\operatorname{Ser}[S]$ & 1 & 12 \\
\hline & & & & & Missense & $\mathrm{C}$ & $\operatorname{Arg}[R]$ & 1 & 12 \\
\hline & & & & & Missense & $\mathrm{T}$ & Cys [C] & 1 & 12 \\
\hline & & & & & Contig reference & $\mathrm{G}$ & Gly [G] & 1 & 12 \\
\hline \multirow[t]{2}{*}{25245352} & 225 & rs397517039 & N.D. & Likely benign & Synonymous & $\mathrm{C}$ & Ala [A] & 3 & 11 \\
\hline & & & & & Contig reference & $\mathrm{T}$ & Ala $[\mathrm{A}]$ & 3 & 11 \\
\hline 25245355 & 221 & rs606231202 & N.D. & Pathogenic & & TGG & Gly [G] & 2 & 10 \\
\hline \multirow[t]{2}{*}{25245361} & 216 & rs 147406419 & 0.001 & other & Synonymous & $\mathrm{G}$ & Val [V] & 3 & 8 \\
\hline & & & & & Contig reference & A & Val [V] & 3 & 8 \\
\hline 25245370 & 207 & rs104894361 & N.D. & Pathogenic & Missense & $\mathrm{T}$ & Asn [N] & 3 & 5 \\
\hline & & & & & Contig reference & $\mathrm{A}$ & Lys [K] & 3 & 5 \\
\hline 25245372 & 205 & rs193929331 & N.D. & Pathogenic & Missense & $\mathrm{G}$ & Glu [E] & 1 & 5 \\
\hline & & & & & Contig reference & $\mathrm{A}$ & Lys [K] & 1 & 5 \\
\hline
\end{tabular}

It's noteworthy to mention that there is (31 SNPs) are synonymous change representing the redundancy of the nucleotide that may be considered as a genetic imprint of Iraqi KRAS gene.

Detailed distribution of SNPs are represented in Fig. 2.

\section{Discussion}

Colorectal cancer poses a great threat of malicious diseases that may appear in human. Most of such cancer cases may be attributed to the diet of people, age, gender and accumulation of genetic changes during aging which may trigger malignant tumors especially in families with such history (Brenner et al.,
2007). Patients consent for this study were divided according to the gender and age. Most cases of colorectal cancer were found in male at elderly age which may be attributed to physiological factors, stress and type of diet, whereas less colorectal cancer were diagnosed in female. No significant malignant tumors were found in younger individuals. For rectal cancer, male and female rates are similar at younger ages, but male rates increasingly predominate at older ages. The frequency of ras mutations to differ significantly with respect to age and sex of patients and location of tumour. This might indicate that there is an association between activation of K-ras and the differences in incidence of colorectal cancer (Breivik et al., 1994). 


\begin{tabular}{llr}
\multicolumn{2}{c}{ Table 4: } & The clinical significance of SNPs found in this study \\
\hline No. & Clinical significance & No. of SNPs \\
\hline 1. & Likely benign & 2 \\
2. & Uncertain significance & 16 \\
3. & Benign & 2 \\
4. & Likely pathogenic & 9 \\
5. & Pathogenic & 22 \\
\hline
\end{tabular}

In addition, degeneration of immune system, diminish of repair system and apoptosis at elderly subjects made them more candidate to colorectal cancer (Levin et al., 2008).

Most of literatures (Bader and Ismail, 2014; Hilmi et al., 2015; Oleg et al., 2015) mentioned that mutation at codon 12 and 13 are more frequent in elderly men than women, that significantly trigger malignant tumor at the colon.

Literatures regarding colorectal cancer in Arabian countries (Wafa and Maher, 2012; Abulkair et al., 2016) studied KRAS mutation at codon 12 and 13 only, but non of these articles detailed the full spectrum of SNPs and their clinical significant as whole.

The study showed that exon 2 at position 5920 located on chromosome 6 may play a vital role in controlling cell cycle and was the main target for genetic changes. Most of missense mutations and frame shift mutations mutations were found at this exon that led consequently to significant change in the resulting protein rendering it with less or no function.

The clinical significance of our finding is listed in Table 4.

\section{Conclusion}

Colorectal cancer is a malicious disease that appear mostly in male than female and was more abundant in elderly subjects. This disease is highly affected by diet and gender and mostly appeared in subjects with family history for malignant tumors. Most of genetic changes were at chromosome 6 position 5920 that included in missense mutation at high rate, frame shift at lower rate. Such accumulative changes led to produce non-functional protein, which stopped apoptosis process in malignant cells causing the tumors to appear in patients.

\section{Ethics Approval and Consent to Participate}

This study did not include any human subjects and was performed on animals postmortem.

\section{Consent for Publication}

This work did not include any personal, written information, pictures and videos to any person.

\section{Availability of Data and Material}

All data and materials used in this study are available and stored at Biotechnology Research Center, Baghdad University and Institut für Virusdiagnostic Reference Laboratory in Germany.

\section{Competing Interests}

This work was conducted without conflict of interest among authors or any other research group in others institutes.

\section{Funding}

This study was performed without any funding from any institute or sponsorship agency.

\section{Authors' Contributions}

All authors contributed to this work according to their major in designing, following, interpreting and data analysis.

\section{References}

Abulkair, O., A. Alqahtani, A. Gasmelseed, N. Abdelhafiz and A. Al Olayan et al., 2016. KRAS mutational status and its clinical implications in saudi colorectal cancer patients. Pan Arab J. Oncol., 9: 12-18.

Arteaga, C.L., 2002. Overview of epidermal growth factor receptor biology and its role as a therapeutic target in human neoplasia. Semin. Oncol., 29: 3-9. DOI: $10.1053 /$ sonc.2002.35642

Bader, T. and A. Ismail, 2014. Higher prevalence of KRAS mutations in colorectal cancer in Saudi Arabia: Propensity for lung metastasis. Alexandria J. Med., 50: 203-209.

DOI: $10.1016 /$ j.ajme.2014.01.003

Botteri, E., S. Iodice, S. Raimondi, P. Maisonneuve and A.B. Lowenfels, 2008. Cigarette smoking and adenomatous polyps: a meta-analysis. Gastroenterology, 134: 388-395. DOI: $10.1053 /$ j.gastro.2007.11.007

Breivik, J., G. Meling, A. Spurkland, T.O. Rognum and G. Gaudernack, 1994. K-ras mutation in colorectal cancer: Relations to patient age, sex and tumour location. Br. J. Cancer, 69: 367-371. DOI: $10.1038 /$ bjc. 1994.67

Brenner, M., M. Hoffmeister, V. Arndt and U. Haug, 2007. Gender differences in colorectal cancer: implications for age at initiation of screening. British J. Cancer, 96: 828-831. DOI: $10.1038 /$ sj.bjc. 6603628 
Castagnola, P. and W. Giaretti, 2005. Mutant KRAS, chromosomal instability and prognosis in colorectal cancer. Biochim. Biophys. Acta, 1756: 115-125. DOI: $10.1016 /$ j.bbcan.2005.06.003

Center, M.M., A. Jemal and E. Ward, 2009. International trends in colorectal cancer incidence rates. Cancer Epidemiol. Biomarkers Prev., 18: 1688-94. DOI: 10.1158/1055-9965.EPI-09-0090

Dyson, J.K. and M.D. Rutter, 2012. Colorectal cancer in inflammatory bowel disease: What is the real magnitude of the risk? World J. Gastroenterol., 18: 3839-384829. DOI: 10.3748/wjg.v18.i29.3839

Giovannucci, E., 2002. Obesity, gender and colon cancer. Gut, 51: 147-147. DOI: $10.1136 /$ gut.51.2.147

Hilmi, K., H. Ilhan, E. Bulent, T. Esam and T. Hilmi et al., 2015. Association between specific KRAS mutations and the clinicopathological characteristics of colorectal tumors. Molecular and Clinical Oncology, 3: 179- 184.

Karapetis, C.S., S. Khambata-Ford, D.J. Jonker, C.J. O'Callaghan and D. Tu et al., 2008. K-ras mutations and benefit from cetuximab in advanced colorectal cancer. N Engl. J. Med., 359: 1757-1765. DOI: $10.1056 /$ NEJMoa0804385
Lee, I.M., E.J. Shiroma, F. Lobelo, P. Puska and S.N. Blair et al., 2012. Effect of physical inactivity on major non-communicable diseases worldwide: an analysis of burden of disease and life expectancy. Lancet, 380: 219-229.

DOI: $10.1016 / \mathrm{S} 0140-6736(12) 61031-9$

Levin, B., D.A. Lieberman, B. McFarland, K.S. Andrews and D. Brooks et al., 2008. Screening and surveillance for the early detection of colorectal cancer and adenomatous polyps, 2008: A joint guideline from the American Cancer Society, the US multi-society task force on colorectal cancer and the American College of Radiology. Gastroenterology, 134: 1570-1595. DOI: 10.1053/j.gastro.2008.02.002

Malumbres, M. and M. Barbacid, 2003. RAS oncogenes: The first 30 years. Nat. Rev. 3: 7-13. DOI: $10.1038 / \mathrm{nrc} 1193$

Oleg, I.K., I.V. Dmitriy, A.G. Yuriy and V. Natalia, 2015. KRAS gene mutations and gender differences in colorectal cancer. Int. J. Biomed., 5: 11-15. DOI: 10.21103/Article5(1)_CR2

Wafa, M. and A. Maher, 2012. KRAS mutations and subtyping in colorectal cancer in Jordanian patients. Oncol. Lett., 4: 705-710. DOI: 10.3892/ol.2012.785 